\title{
Oxygen Modulates Prostacyclin Synthesis in Ovine Fetal Pulmonary Arteries by an Effect on Cyclooxygenase
}

Philip W. Shaul, William B. Campbell, ${ }^{*}$ Melody A. Farrar, and Ronald R. Magness

Departments of Pediatrics and *Pharmacology, University of Texas Southwestern Medical Center at Dallas, Dallas, Texas 75235-9063

\begin{abstract}
Prostacyclin ( $\mathrm{PGI}_{2}$ ) plays an integral role in $\mathrm{O}_{2}$ mediation of pulmonary vasomotor tone in the fetus and newborn. We hypothesized that $\mathrm{O}_{2}$ modulates $\mathrm{PGI}_{2}$ synthesis in vitro in ovine fetal intrapulmonary arteries, with decreasing $\mathrm{O}_{2}$ causing attenuated synthesis. A decline in $\mathrm{Po}_{2}$ from 680 to $40 \mathrm{mmHg}$ caused a $26 \%$ fall in basal PGI $_{2}$ synthesis. PGI $_{2}$ synthesis maximally stimulated by bradykinin, A23187, and arachidonic acid were also attenuated at low $\mathrm{Po}_{2}$, by $35 \%, 33 \%$, and 35\%, respectively. $\mathbf{P G E}_{2}$ synthesis was equally affected. In contrast, varying $\mathrm{O}_{2}$ did not alter $\mathbf{P G I}_{2}$ synthesis with exogenous $\mathbf{P G H}_{2}$, which is the product of cyclooxygenase and the substrate for prostacyclin synthetase. Prostaglandin-mediated effects of $\mathrm{O}_{2}$ on cAMP production were also examined. Decreasing $\mathrm{Po}_{2}$ to $40 \mathrm{mmHg}$ caused complete inhibition of basal cAMP production, whereas cAMP production stimulated by exogenous $\mathrm{PGI}_{2}$ was not affected. In parallel studies of mesenteric arteries, $\mathbf{P G I}_{2}$ synthesis and cAMP production were enhanced at low $\mathrm{O}_{2}$. Thus, $\mathbf{P G I}_{2}$ synthesis in fetal intrapulmonary arteries is modulated by changes in $\mathrm{O}_{2}$, with decreasing $\mathrm{O}_{2}$ causing attenuated synthesis. This process is due to an effect on cyclooxygenase activity, it causes marked parallel alterations in cAMP production, and it is specific to the pulmonary circulation. ( $J$. Clin. Invest. 1992. 90:2147-2155.) Key words: adenylate cyclase - cyclic adenosine monophosphate - hypoxic pulmonary hypertension • mesenteric artery $\bullet$ prostaglandin $\mathbf{E}_{2}$
\end{abstract}

\section{Introduction}

Oxygen is an important mediator of pulmonary vasomotor tone in the fetus and newborn, with hyperoxia and hypoxia causing pulmonary vasodilatation and vasoconstriction, respectively (1-4). The response of the pulmonary vascular bed contrasts with that of the systemic circulation, as the latter dilates with decreased oxygenation (1-3). These physiologic responses are critically involved in the normal transition of the pulmonary circulation from that of the fetus (high resistance) to that of the newborn (low resistance), and they have also been implicated in the pathophysiology of persistent pulmonary hypertension of the newborn (PPHN), a condition associated with both high morbidity and a mortality rate of 12 $50 \%(5-8)$.

Address reprint requests to Dr. Shaul, Department of Pediatrics, University of Texas Southwestern Medical Center at Dallas, 5323 Harry Hines Blvd., Dallas, TX 75235-9063.

Received for publication 26 February 1992 and in revised form 8 June 1992.

J. Clin. Invest.

(c) The American Society for Clinical Investigation, Inc.

0021-9738/92/12/2147/09 \$2.00

Volume 90, December 1992, 2147-2155
Pioneering studies in the fetal lamb have demonstrated that enhanced oxygenation of one lung in situ leads to decreased vascular resistance solely in that lung, indicating that oxygenrelated responses in the pulmonary circulation are locally mediated (9). There is substantial evidence in the lamb and in other animal models that vasodilatory prostaglandins such as prostacyclin and prostaglandin $\mathrm{E}_{2}\left(\mathrm{PGE}_{2}\right)$ may play an integral role in these processes. The prostanoids have been implicated because: $(a)$ they are potent dilators of the pulmonary circulation of the fetus and newborn when administered exogenously $(10,11) ;(b)$ they are produced by the lungs during the transition of the pulmonary circulation at birth, which occurs at least in part in response to increased oxygenation $(12,13)$; and (c) inhibition of prostaglandin synthesis attenuates the normal decline in pulmonary vascular resistance during transition (14, 15 ). Pulmonary prostaglandins are primarily produced by the vascular cells, particularly the endothelium (16).

To better understand the mechanism(s) underlying oxygen modulation of vasomotor tone in the developing pulmonary circulation, the present study was designed to determine the effects of acute changes in in vitro oxygenation on prostacyclin synthesis in intact intrapulmonary arteries obtained from late gestation ovine fetuses. Based on the in vivo findings indicating enhanced vasodilatory prostaglandin production by the fetal lungs under conditions of increased oxygenation (12-15), the hypothesis was put forth that oxygen modulates prostacyclin synthesis in the intrapulmonary arteries, with decreased oxygenation causing attenuated synthesis. In addition to testing this hypothesis, studies were performed to address the following questions: $(a)$ Is the effect of alterations in oxygenation on prostacyclin synthesis specific to the intrapulmonary arteries? (b) What step (s) in the synthetic cascade is involved in oxygen modulation of prostacyclin synthesis in the intrapulmonary arteries? and $(c)$ Do changes in oxygenation modify prostaglandin-mediated signal transduction in the vascular smooth muscle (VSM), ${ }^{1}$ specifically cAMP production?

\section{Methods}

Animal model. The lamb has been used extensively in investigations examining physiologic parameters to assess the role of vasodilatory prostaglandins in oxygen modulation of vascular resistance in the developing pulmonary circulation (10-14). As such, it is an excellent animal model for the in vitro study of the effects of oxygen on pulmonary artery prostacyclin synthesis in the fetus. In the present investigation, intrapulmonary arterial segments were obtained from mixed breed fetal lambs at $125-135 \mathrm{~d}$ of gestation $(n=52)$, with term being $144 \pm 4 \mathrm{~d}$. The pregnant ewes utilized $(n=27)$ were multiparous and had singletons $(n=7)$, twins $(n=15)$, or triplets $(n=5)$. In selected experiments, mesenteric arteries were also studied as representative systemic arteries. Before sacrifice the ewes were housed in the Animal Resources

1. Abbreviations used in this paper: IBMX, isobutylmethylxanthine; VSM, vascular smooth muscle. 
Center of the University of Texas Southwestern Medical Center and were given standard laboratory animal diet and water ad libitum. The procedures followed in the care and euthanasia of the study animals were approved by the Institutional Review Board for Animal Research.

Arterial segment preparation. The procedures used generally follow those we have previously reported $(17,18)$. Briefly, the ewe and fetus(es) were euthanized with sodium pentobarbital $(50 \mathrm{mg} / \mathrm{kg})$ given intravenously to the ewe, and the fetuses were delivered by cesarean section. The fetal lungs and the mesentery containing the cephalic mesenteric arterial cascade were immediately removed and placed in ice cold phosphate-buffered saline $\left(0.01 \mathrm{M} \mathrm{PO}_{4}, 0.15 \mathrm{M} \mathrm{NaCl}, \mathrm{pH}\right.$ 7.4). Further tissue preparation was performed in a cold room at $4^{\circ} \mathrm{C}$. The pulmonary arterial tree was rapidly dissected from the lung parenchyma and the mesenteric arterial bed was isolated from the surrounding tissue, and both were placed in fresh ice cold phosphate-buffered saline. Remaining fatty and connective tissue was removed and the adventitia was grossly dissected from the arteries, taking care not to disrupt the endothelium.

Fourth-generation intrapulmonary arteries (19) and mesenteric arteries of similar size (0.5-1 mm o.d.) were isolated and placed in freshly prepared Krebs-Henseleit buffer gassed with $95 \% \mathrm{O}_{2} / 5 \% \mathrm{CO}_{2}$ at $37^{\circ} \mathrm{C}$. The Krebs-Henseleit buffer contained $4.8 \mathrm{mM} \mathrm{KCl}, 2.0 \mathrm{mM} \mathrm{CaCl}_{2}, 1.2$ $\mathrm{mM} \mathrm{KH}_{2} \mathrm{PO}_{4}, 1.2 \mathrm{mM} \mathrm{MgSO}_{4}, 11.0 \mathrm{mM}$ dextrose, $118 \mathrm{mM} \mathrm{NaCl}$, and $25 \mathrm{mM} \mathrm{NaHCO}_{3}$ at $\mathrm{pH}$ 7.4. Fourth-generation intrapulmonary arteries were chosen for study in an effort to examine these biochemical mechanisms in freshly obtained intact arterial segments as close to the level of the resistance vessels as possible (19). The mesenteric arteries were selected for study because the ovine fetal mesenteric circulation typifies the variety of systemic vascular beds that are responsive in vivo to exogenous vasodilatory prostaglandins (20) and inhibitors of prostaglandin synthesis (21). Segments with wet weights of 1-4 mg were cut, rinsed in fresh oxygenated Krebs-Henseleit buffer, and equilibrated in the oxygenated buffer at $37^{\circ} \mathrm{C}$ for $2 \mathrm{~h}$. The presence of intact, functional endothelium was confirmed in randomly chosen segments by (a) light microscopy of $5-\mu$ m sections of the arteries, $(b)$ examinations of endothelium-dependent relaxation with acetylcholine; and $(c)$ quantification of cGMP production with acetylcholine stimulation (18).

Incubations for prostaglandin synthesis. The incubation procedures employed were similar to those we have used in studies of uterine and systemic arteries from pregnant sheep (22). After collective equilibration, the segments were placed individually into sealed polypropylene chambers containing $2.0 \mathrm{ml}$ of oxygenated $(680 \mathrm{mmHg})$ KrebsHenseleit buffer at $37^{\circ} \mathrm{C}$ for $1 \mathrm{~h}$. At the end of this preincubation period, the media was replaced with fresh Krebs-Henseleit buffer and a 5-20-min incubation was performed. At the end of the incubation, the media was placed into ice cold tubes containing $100 \mu \mathrm{g}$ of acetylsalicylic acid and was stored at $-20^{\circ} \mathrm{C}$ until the time of assay for prostaglandins. The segments were placed in ice-cold $7 \%$ trichloroacetic acid (TCA) and were stored at $-20^{\circ} \mathrm{C}$ until protein content was determined by a modification of the method of Lowry et al. (23) using bovine serum albumin as the standard. It was demonstrated in initial studies that under these conditions the prostanoids measured are newly synthesized, and prostacyclin synthesis is linear with time for up to $2 \mathrm{~h}$.

To assess oxygen modulation of prostacyclin synthesis, the incubations were conducted at three levels of in vitro oxygenation. The incubation media was bubbled with one of three gas mixtures both before and after the placement of the media into the sealed chambers containing the preincubated arterial segments. The resulting $\mathrm{pH}, \mathrm{PCO}_{2}$, and $\mathrm{PO}_{2}$ in the incubation media were determined with Micro 13 Blood Gas Analyzer (Instrumentation Laboratory, Inc., Lexington, MA). Gassing with $95 \% \mathrm{O}_{2} / 5 \% \mathrm{CO}_{2}$ yielded a $\mathrm{PO}_{2}$ of $678-680 \mathrm{mmHg}$, gassing with $20 \% \mathrm{O}_{2} / 5 \% \mathrm{CO}_{2} / 75 \% \mathrm{~N}_{2}$ resulted in a $\mathrm{Po}_{2}$ of $150-155 \mathrm{mmHg}$, and the use of $0 \% \mathrm{O}_{2} / 5 \% \mathrm{CO}_{2} / 95 \% \mathrm{~N}_{2}$ gave a $\mathrm{PO}_{2}$ in the incubation media of $40-42 \mathrm{mmHg}$. The purpose of selecting these levels of $\mathrm{PO}_{2}$ in the incubation media was to provide a wide spectrum of in vitro oxygenation, and not to necessarily mimic levels of oxygenation that yield cardiovascular effects in vivo. The use of $5 \% \mathrm{CO}_{2}$ maintained the $\mathrm{PCO}_{2}$ of the incubation media at $36-44 \mathrm{mmHg}$ and the $\mathrm{pH}$ at $7.35-7.44$.
In experiments determining the step(s) in the synthetic cascade which is involved in oxygen modulation of prostacyclin synthesis, some arterial segments were incubated in Krebs-Henseleit buffer alone, indicative of basal (nonstimulated) synthesis, and others were incubated with agents that activate prostacyclin synthesis by specific mechanisms. Incubations were performed in the presence of bradykinin to assess the effects of oxygen on prostacyclin synthesis stimulated by an agonist that activates arachidonic acid mobilization from phospholipids via a receptor-mediated mechanism (24). Reversal of the oxygen effect would be seen if the effect involved alterations in the production of a local agonist such as bradykinin, as has been suggested by Fineman et al. (25). Incubations with the calcium ionophore A23187 were performed to determine the effects of oxygen on prostacyclin synthesis stimulated by an increase in cytosolic calcium, which activates arachidonic acid mobilization by a nonreceptor-mediated process (26). If the oxygen effect was due to changes in calcium-related mechanisms mediating arachidonic acid mobilization, one would expect the effect to be negated by $\mathrm{A} 23187$ treatment. Exogenous arachidonic acid was also used to stimulate prostacyclin synthesis to reveal if the effect of oxygen is reversed by provision of the precursor (26). Reversal of the oxygen effect would be seen if the effect occurred proximal to arachidonic acid in the synthetic cascade, and a lack of reversal would indicate that the mechanism involves changes in cyclooxygenase and/or prostacyclin synthetase activity (26). Lastly, incubations were done in the presence of exogenous $\mathrm{PGH}_{2}$, the product of cyclooxygenase activity and the substrate for prostacyclin synthetase, to differentiate between potential effects on the activities of these enzymes (26). Studies of dose responses and time courses of activation of the synthetic cascade preceeded the experiments at varying levels of oxygenation to ensure that maximal stimulation was examined, and that stimulated prostacyclin synthesis was linear with time over the duration of incubation.

Samples of incubation media were assayed for the stable metabolite of prostacyclin, 6-keto- $\mathrm{PGF}_{1 \alpha}$ by radioimmunoassay as previously reported (27). Briefly, the 6-keto-PGF ${ }_{1 \alpha}$ assay procedure utilized duplicate aliquots of standard (0-200 pg) and incubation samples put into a mixture of Krebs-Henseleit solution and $0.1 \mathrm{M}$ phosphate-buffered saline (1:1) plus $0.1 \%$ polyvinyl-pyrrolidone. Antiserum $(0.1 \mathrm{ml}$, 1:4,000 titer $)$ and $0.1 \mathrm{ml}$ [ $\left.{ }^{3} \mathrm{H}\right]$ 6-keto-PGF ${ }_{1 \alpha}(12,000 \mathrm{dpm})$ were added and the tubes were incubated at $4^{\circ} \mathrm{C}$ for $12-18 \mathrm{~h}$. Bound and free ligand were separated with dextran-coated charcoal and bound ligand was counted by liquid scintillation spectrometry. In selected experiments, incubation media was also assayed for $\mathrm{PGE}_{2}$ by radioimmunoassay as previously reported (27). For the $\mathrm{PGE}_{2}$ assay duplicate aliquots of standard (0-500 pg) and incubation samples were placed in the same mixture of Krebs-Henseleit solution and phosphate-buffered saline. Antiserum (0.1 ml, 1:14,000 titer) and $0.1 \mathrm{ml}\left[{ }^{3} \mathrm{H}\right] \mathrm{PGE}_{2}$ ( 16,500 dpm) were added and the tubes were incubated, bound and free ligand were separated, and bound ligand was quantified as in the 6-keto-PGF $F_{1 \alpha}$ assay. The unknown quantities of 6-keto-PGF ${ }_{1 \alpha}$ and $\mathrm{PGE}_{2}$ were determined from the standard curves generated. The intra- and interassay coefficients of variation in the 6-keto-PGF ${ }_{1 \alpha}$ assay were $5.8 \%$ and $8.9 \%$ at $250 \mathrm{pg} / \mathrm{ml}$, and $2.8 \%$ and $8.7 \%$ at $1,000 \mathrm{pg} / \mathrm{ml}$. For $\mathrm{PGE}_{2}$ they were $10.8 \%$ and $13.8 \%$ at $250 \mathrm{pg} / \mathrm{ml}$, and $7.0 \%$ and $17.7 \%$ at $1,000 \mathrm{pg} / \mathrm{ml}$.

Incubations for cAMP production. Cyclic AMP production was measured in the arterial segments to determine if the effects of alterations in oxygenation on prostacyclin synthesis are sufficient to modify the second messenger system through which prostacyclin has its action (27). The cAMP contents measured are the summation of cAMP in both the endothelial and VSM cells. Adenylate cyclase activity has been demonstrated in endothelial cells, but comparisons of enzyme activity in endothelial and VSM cells cultured from the same artery indicate that activity is much greater in the VSM (28). Furthermore, the vast majority of the cells examined in the present study are VSM, and as a result the cAMP measured primarily represents VSM cAMP.

The techniques used are as previously described. Cyclic AMP production is determined by measuring the accumulation of the cyclic nucleotide in the segments during incubations in the presence of phosphodiesterase inhibition (17). After collective equilibration, segments 
for cAMP production were placed individually into polypropylene chambers containing $1.0 \mathrm{ml}$ of oxygenated $(680 \mathrm{mmHg}) \mathrm{Krebs}$-Henseleit buffer for a 20 -min preincubation at $37^{\circ} \mathrm{C}$. The cAMP content of a selected number of these nontreated segments was determined at the end of the preincubation period. The preincubation media was aspirated from the tubes, $0.5 \mathrm{ml}$ of ice-cold $7 \%$ TCA was added to the tubes containing the arterial segments, and they were placed on ice. The segments were frozen in TCA until homogenization and the determination of cAMP content were performed.

Cyclic AMP production due to adenylate cyclase stimulation by endogenous agonists (herein referred to as basal cAMP production) was determined in a separate set of segments. After preincubation, the segments were incubated for $10 \mathrm{~min}$ in fresh Krebs-Henseleit buffer in the presence of the phosphodiesterase inhibitor isobutylmethylxanthine (IBMX, $\left.10^{-4} \mathrm{M}\right)(17)$. The incubations were begun by replacing the preincubation media with fresh IBMX-containing media that had been continuously bubbled with one of the three gas mixtures. The gassing of the media was also resumed immediately after it was placed into the chamber containing the preincubated arterial segment. At the end of the incubation period, the media was removed and the segments were placed in 7\% TCA and stored as described above. Preliminary studies revealed that the addition of IBMX does not alter prostaglandin synthesis in these arteries. In one series of experiments, selected intrapulmonary segments were incubated in the presence of IBMX plus 100 $\mu \mathrm{M}$ indomethacin to determine the role of endogenous prostaglandins in the mediation of basal cAMP production. Indomethacin at this concentration inhibits prostaglandin synthesis in these arteries by $88 \%$. Additional segments were treated with IBMX and indomethacin, plus either $10^{-5} \mathrm{M}$ prostacyclin or $10^{-5} \mathrm{M} \mathrm{PGE}_{2}$ to determine if the effect of decreased prostaglandin synthesis on cAMP production is reversed by exogenous agonist.

Cyclic AMP production with exogenous stimulation of adenylate cyclase by prostacyclin $\left(10^{-5} \mathrm{M}\right)$ was also examined at the three levels of oxygenation during incubations of the segments with IBMX. This was done to determine if the responsiveness of adenylate cyclase to prostacyclin differs with varying oxygenation. To evaluate whether such a potential change in enzyme sensitivity is specific to the prostanoids, $\beta$-adrenergic receptor-mediated cAMP production was measured during $10 \mathrm{~min}$ incubations with $10^{-5} \mathrm{M}$ isoproterenol (17). In addition, cAMP production with postreceptor-mediated stimulation of adenylate cyclase was assessed by quantifying the increase in cAMP content during 10 -min incubations in the presence of $10^{-4} \mathrm{M}$ forskolin (17). The experiments with isoproterenol and forskolin were performed exclusively in intrapulmonary segments because of the limited number of segments available from the mesenteric arteries.

The cAMP contents of nontreated and treated arterial segments were determined after thawing on ice and homogenization in a ground glass homogenizer using a motorized pestle (17). Duplicate aliquots of homogenate were set aside for protein determination by the method described above (23). The remainder of the homogenate was centrifuged at $4^{\circ} \mathrm{C}$ at $1,300 \mathrm{~g}$ for $15 \mathrm{~min}$ and an ether extraction was performed on $50 \mu \mathrm{l}$ of the supernatant $(10 \times$ volume, $\times 3)$. The ether-extracted aliquot was dried at $40^{\circ} \mathrm{C}$ for 12 to $16 \mathrm{hr}$ and cAMP content was determined by radioimmunoassay following acetylation $(17,27,29)$. Recovery was generally $>87 \%$. The intraassay and interassay coeffcients of variation for the cAMP radioimmunoassay were $3.6 \%$ and $7.8 \%$, respectively.

Statistical analysis. Analysis of variance (ANOVA) with NeumanKeuls post-hoc testing was employed to compare mean values between more than two groups. Nonparametric ANOVA was used when indicated. Single comparisons between two groups were performed with paired and nonpaired Student's $t$ tests. Significance was accepted at the 0.05 level of probability. All results are expressed as mean \pm SEM.

Materials. Prostacyclin (sodium salt) and $\mathrm{PGE}_{2}$ were obtained from The Upjohn Co. (Kalamazoo, MI). Prostacyclin was dissolved in $50 \mathrm{mM}$ Tris buffer, $\mathrm{pH}$ 9.7, and $\mathrm{PGE}_{2}$ was dissolved in $0.1 \mathrm{M} \mathrm{PO}_{4}$ buffer, $\mathrm{pH}$ 7.4. 6-Keto-PGF ${ }_{1 \alpha}$ standard was from Cayman Chemical Co. (Ann Arbor, MI). $\left[{ }^{3} \mathrm{H}\right] 6-\mathrm{Keto}^{-} \mathrm{PGF}_{1 \alpha}(120-180 \mathrm{Ci} / \mathrm{mmol})$ and $\left[{ }^{3} \mathrm{H}\right] \mathrm{PGE}_{2}(140-170 \mathrm{Ci} / \mathrm{mmol}$ ) were from Amersham Corp. (Arlington Heights, IL). Bradykinin (acetate salt), A23187 (hemimagnesium salt), arachidonic acid (sodium salt), L-isoproterenol (D-bitartrate salt), and forskolin were obtained from Sigma Chemical Co. (St. Louis, MO). Forskolin was initially dissolved in $95 \%$ ethanol to yield a $10 \mathrm{mM}$ solution and further dilutions were with Krebs-Henseleit buffer. The amount of ethanol present in the incubations with forskolin $(<1 \%)$ had no effect on segment cAMP content when tested alone. $\mathrm{PGH}_{2}$ was from Biomol Research Laboratories, Inc. (Plymouth Meeting, PA); the hexane-ethyl acetate vehicle (10:1) was rapidly evaporated over $\mathrm{N}_{2}$ and the compound was placed in Krebs-Henseleit buffer immediately before use. All solutions were prepared fresh daily. Cyclic AMP radioimmunoassay kits were from New England Nuclear/Dupont (Boston, MA).

\section{Results}

Basal prostacyclin synthesis. The effects of alterations in in vitro oxygenation on basal prostacyclin synthesis in the intrapulmonary and systemic arteries are shown in Fig. 1. At $\mathrm{PO}_{2}$ $680 \mathrm{mmHg}$, prostacyclin synthesis was similar in the two artery types. With a decline in $\mathrm{PO}_{2}$ to $150 \mathrm{mmHg}$, there was no signifcant change in prostacyclin production in either artery. However, a decline in $\mathrm{PO}_{2}$ to $40 \mathrm{mmHg}$ resulted in $26 \%$ attenuation of prostacyclin synthesis in the intrapulmonary segments, and this was contrasted by an increase in synthesis in the systemic segments of $96 \%$.

Stimulated prostacyclin synthesis. To define the step(s) in the synthetic cascade which is involved in oxygen modulation of prostacyclin synthesis in the intrapulmonary arteries, the effects of alterations in oxygenation on stimulated prostacyclin synthesis were determined. This was preceeded by studies at $\mathrm{PO}_{2} 680 \mathrm{mmHg}$ of dose responses and time courses of stimulation of the synthetic cascade. The maximal responses to bradykinin and to $\mathrm{A} 23187$ were achieved at concentrations of $10^{-6}$ M (Fig. 2, $A$ and $B$, respectively). Maximal stimulation with arachidonic acid was obtained at $10^{-5} \mathrm{M}$ (Fig. $2 \mathrm{C}$ ). Prostacyclin synthesis with the addition of exogenous $\mathrm{PGH}_{2}$ was examined at $10^{-6}$ and $10^{-5} \mathrm{M}$ only, with 2.1 -fold greater prostacyclin synthesis at the higher concentration (Fig. $2 D$ ). As a result of these findings, ensuing studies of stimulated prostacyclin

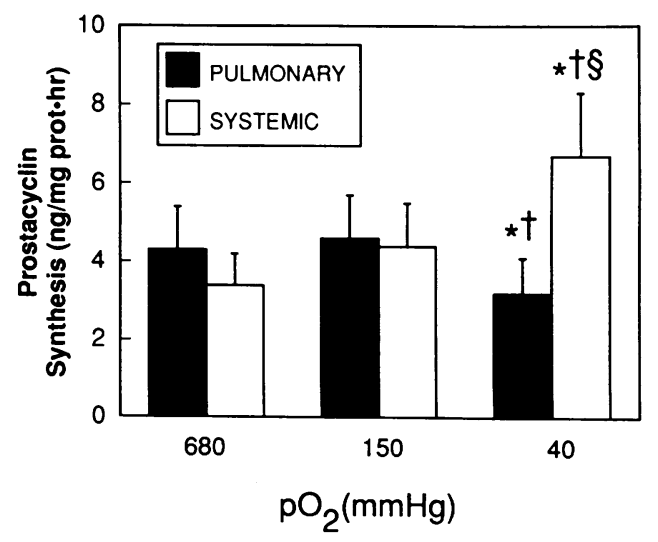

Figure 1. Effect of oxygen tension on prostacyclin synthesis in pulmonary (solid bars) and systemic arterial segments (open bars). The segments were incubated for $20 \mathrm{~min}$ and prostacyclin (6-keto PGF $_{1 \propto}$ ) production was measured by radioimmunoassay. Values are mean \pm SEM for duplicate segments from 12 fetal lambs. ${ }^{*} P<0.05$ vs. $680 \mathrm{mmHg},{ }^{\dagger} P<0.05$ vs. $150 \mathrm{mmHg},{ }^{\S} P<0.05$ vs. pulmonary. 

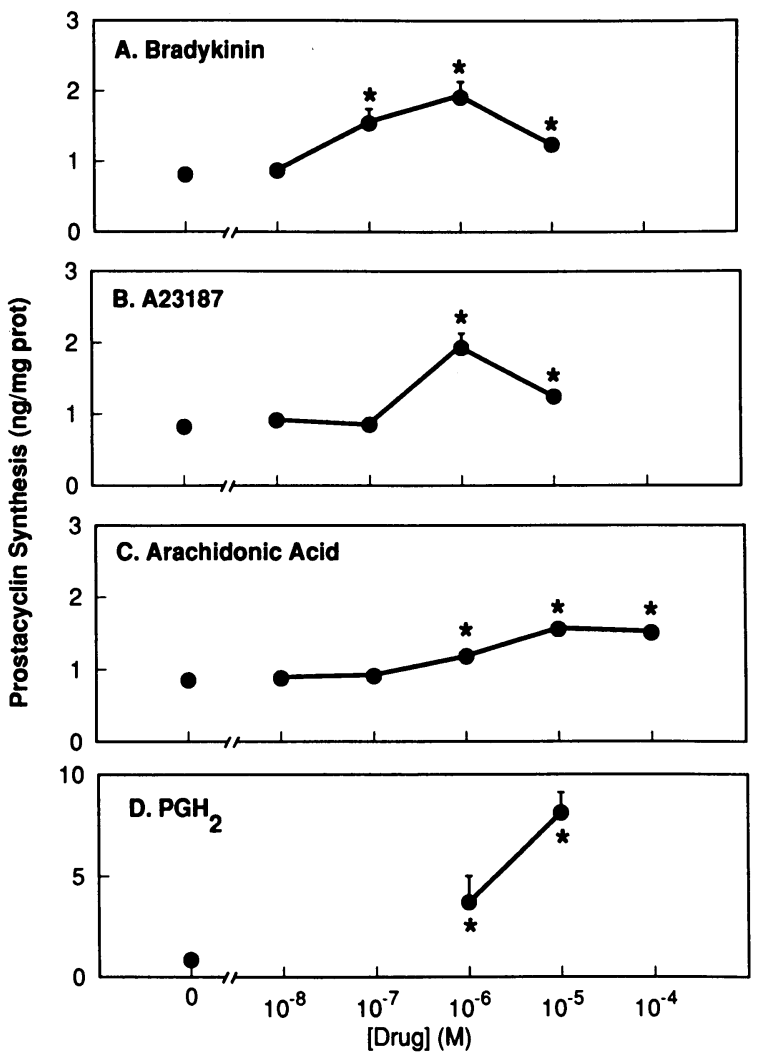

Figure 2. Dose-response studies of prostacyclin synthesis stimulated by $(A)$ bradykinin, $(B) \mathrm{A} 23187,(C)$ arachidonic acid, and $(D)$ $\mathrm{PGH}_{2}$. Pulmonary arterial segments were incubated at $\mathrm{PO}_{2} 680$ $\mathrm{mmHg}$ for $10 \mathrm{~min}$ (bradykinin, A23187, and arachidonic acid) or for $5 \mathrm{~min}\left(\mathrm{PGH}_{2}\right)$, and prostacyclin (6-keto $\left.\mathrm{PGF}_{1 \propto}\right)$ production was measured by radioimmunoassay. Values are mean \pm SEM for duplicate segments from two to five fetal lambs. For some data points the error bars are smaller than the symbols depicting the mean values. Note the differences in the scales in $A-C$ compared to $D$. ${ }^{*} P<0.05$ vs. no treatment.

synthesis were performed with $10^{-6} \mathrm{M}$ bradykinin, $10^{-6} \mathrm{M}$ A23187, $10^{-5} \mathrm{M}$ arachidonic acid, or $10^{-5} \mathrm{M} \mathrm{PGH}_{2}$.

The time courses for basal and stimulated prostacyclin synthesis are depicted in Fig. 3. Basal prostacyclin synthesis was linear with time up to at least $15 \min (r=0.99, P<0.001$, Fig. $3 A)$, as was synthesis stimulated with bradykinin $(r=0.96, P$ $<0.04$, Fig. $3 \mathrm{~B}$ ). Prostacyclin synthesis in the presence of A23187 was linear with time up to $10 \min (r=0.99, P<0.04)$ and it did not increase thereafter (Fig. $3 C$ ). The response to exogenous arachidonic acid was linear with time up to at least $15 \min (r=0.99, P<0.02$, Fig. $3 \mathrm{D})$, and synthesis in the presence of exogenous $\mathrm{PGH}_{2}$ was linear with time for at least $10 \min (r=0.99, P<0.02$, Fig. $3 E)$. As a result of these observations, the studies of stimulated prostacyclin synthesis at varying levels of oxygenation were $10 \mathrm{~min}$ in duration for all treatments except $\mathrm{PGH}_{2}$, which entailed a 2-min incubation.

The effects of alterations in in vitro oxygenation on intrapulmonary artery prostacyclin and $\mathrm{PGE}_{2}$ synthesis with bradykinin stimulation are shown in Fig. 4. Oxygen-modulated changes in basal prostanoid synthesis were studied in parallel in these experiments. Similar to the findings in the previous experiments (Fig. 1), basal prostacyclin synthesis ( Fig. $4 A$ ) was equivalent in segments studied at $\mathrm{PO}_{2}$ levels of 680 and 150
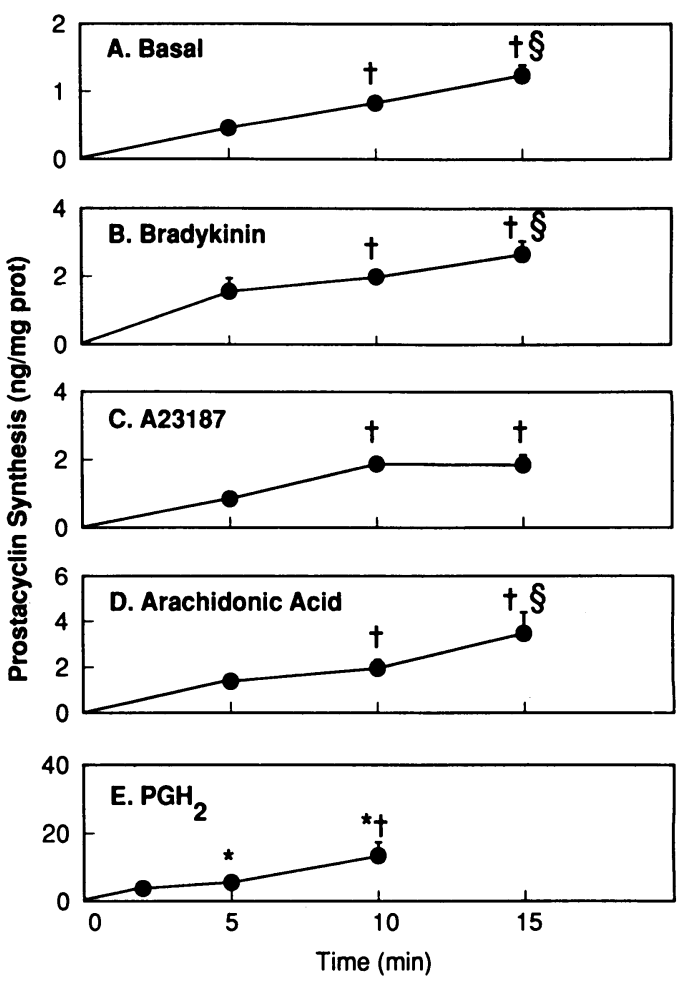

Figure 3. Time courses for basal and stimulated prostacyclin synthesis. Pulmonary arterial segments were incubated at $\mathrm{Po}_{2} 680 \mathrm{mmHg}$ in the absence of treatment (basal, $A$ ) or in the presence of $10^{-6} \mathrm{M}$ bradykinin $(B), 10^{-6} \mathrm{M} \mathrm{A23187}(C), 10^{-5} \mathrm{M}$ arachidonic acid $(D)$, or $10^{-5} \mathrm{M} \mathrm{PGH}_{2}(E)$. Prostacyclin (6-keto $\mathrm{PGF}_{1 \propto}$ ) production was measured by radioimmunoassay. Values are mean \pm SEM for duplicate segments from four fetal lambs. For some data points the error bars are smaller than the symbols depicting the mean values. Note the differences in the scales in $A, B$ and $C, D$, and $E{ }^{*} P<0.05$ vs. 2 min, ${ }^{\dagger} P<0.05$ vs. $5 \mathrm{~min},{ }^{\S} P<0.05$ vs. $10 \mathrm{~min}$.

$\mathrm{mmHg}$, but it was attenuated $26 \%$ at $\mathrm{Po}_{2} 40 \mathrm{mmHg}$. Basal $\mathrm{PGE}_{2}$ synthesis (Fig. $4 \mathrm{~B}$ ), which was $69 \%$ greater than prostacyclin synthesis at $680 \mathrm{mmHg}$ in these experiments, was also not altered at $\mathrm{PO}_{2} 150 \mathrm{mmHg}$. However, in parallel to the find-

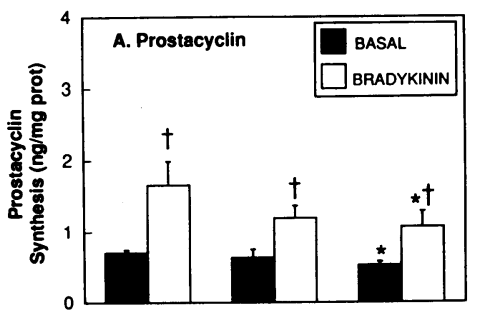

Figure 4. Effect of oxygen tension on bradykinin-stimulated $(A)$ prostacyclin and $(B) \mathrm{PGE}_{2}$ synthesis. Pulmonary arterial segments were incubated for $10 \mathrm{~min}$ in the absence of treatment (basal,

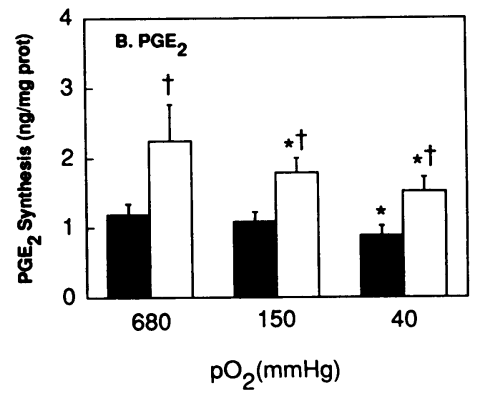
solid bars) or in the presence of $10^{-6} \mathrm{M}$ bradykinin (open bars). The amount of prostacyclin (6-keto $\left.\mathrm{PGF}_{1 \alpha}\right)$ and $\mathrm{PGE}_{2}$ produced was measured by radioimmunoassay. Values are mean \pm SEM for duplicate segments from six fetal lambs. ${ }^{*} P<0.05$ vs. 680 $\mathrm{mmHg},{ }^{\dagger} P<0.05$ vs. basal. 

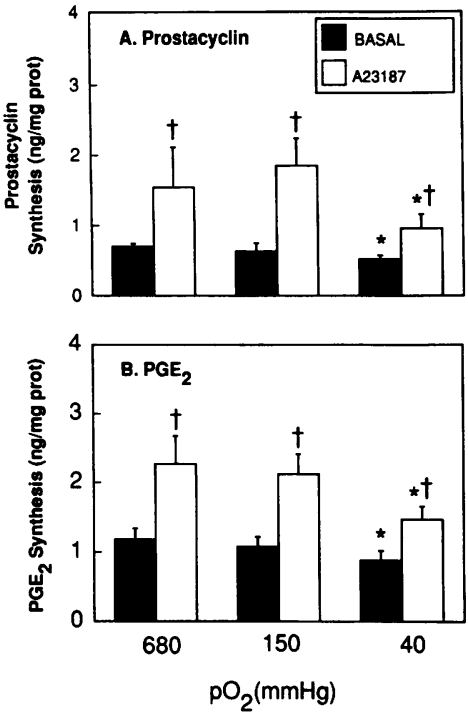

Figure 5. Effect of oxygen tension on A23187-stimulated $(A)$ prostacyclin and (B) $\mathrm{PGE}_{2}$ synthesis. Pulmonary arterial segments were incubated for $10 \mathrm{~min}$ in the absence of treatment (basal, solid bars) or in the presence of $10^{-6} \mathrm{M} \mathrm{A23187}$ (open bars). The amount of prostacyclin (6-keto $\left.\mathrm{PGF}_{1 \propto}\right)$ and $\mathrm{PGE}_{2}$ produced was measured by radioimmunoassay. Values are mean \pm SEM for duplicate segments from six fetal lambs. ${ }^{*} P<0.05$ vs. 680 $\mathrm{mmHg},{ }^{\dagger} P<0.05$ vs. basal. ings for basal prostacyclin synthesis, basal $\mathrm{PGE}_{2}$ synthesis was decreased by $25 \%$ at $40 \mathrm{mmHg}$. Bradykinin-stimulated prostacyclin synthesis (Fig. $4 \mathrm{~A}$ ) was also attenuated at lower $\mathrm{PO}_{2}$, with statistical significance being reached at $\mathrm{PO}_{2} 40 \mathrm{mmHg}$, at which synthesis was decreased by $35 \%$ compared to at $\mathrm{Po}_{2} 680$ mmHg. Concomitantly, bradykinin-stimulated $\mathrm{PGE}_{2}$ synthesis (Fig. $4 \mathrm{~B}$ ) was also attenuated at lower $\mathrm{PO}_{2}$, by $20 \%$ and $32 \%$ at 150 and $40 \mathrm{mmHg}$, respectively.

The effects of changes in oxygenation on intrapulmonary artery prostacyclin and $\mathrm{PGE}_{2}$ synthesis with $\mathrm{A} 23187$ stimulation are shown in Figs. 5, $A$ and $B$, respectively. Prostacyclin synthesis with $A 23187$ was similar at 680 and $150 \mathrm{mmHg}$, but was decreased $33 \%$ at $\mathrm{PO}_{2} 40 \mathrm{mmHg}$. Similarly, $\mathrm{PGE}_{2}$ synthesis in the presence of the calcium ionophore was not altered at 150 $\mathrm{mmHg}$, but it was attenuated by $35 \%$ at $40 \mathrm{mmHg}$.

Prostacyclin and $\mathrm{PGE}_{2}$ synthesis with arachidonic acid at the three levels of oxygenation are shown in Fig. 6, $A$ and $B$, respectively. Prostacyclin synthesis with a maximally stimulating concentration of the substrate was decreased at lower $\mathrm{PO}_{2}$, reaching statistical significance at $\mathrm{PO}_{2} 40 \mathrm{mmHg}$, at which it was attenuated by $35 \%$ compared to at $\mathrm{Po}_{2} 680 \mathrm{mmHg}$. In
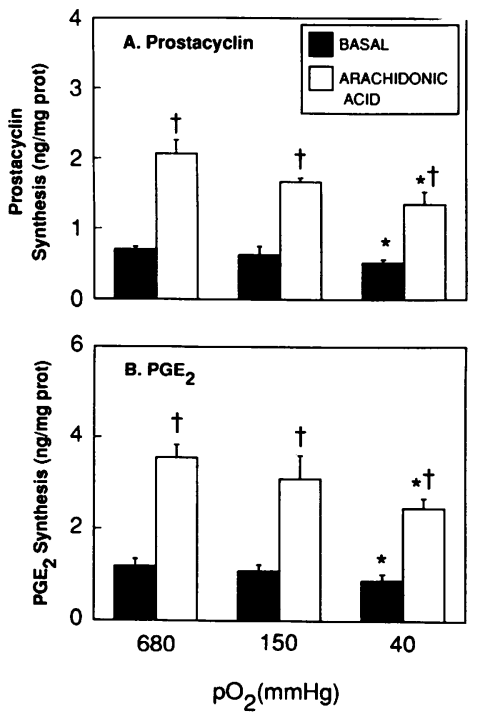

Figure 6. Effect of oxygen tension on arachidonic acid-stimulated $(A)$ prostacyclin and $(B) \mathrm{PGE}_{2}$ synthesis. Pulmonary arterial segments were incubated for $10 \mathrm{~min}$ in the absence of treatment (basal, solid $10^{-5} \mathrm{M}$ arachidonic acid (open bars). The amount of prostacyclin (6-keto $\left.\mathrm{PGF}_{1 \propto}\right)$ and $\mathrm{PGE}_{2}$ produced was measured by radioimmunoassay. Values are mean \pm SEM for duplicate segments from six fetal lambs. ${ }^{*} P<0.05$ vs. 680 $\mathrm{mmHg},{ }^{\dagger} P<0.05$ vs. basal. bars) or in the presence of
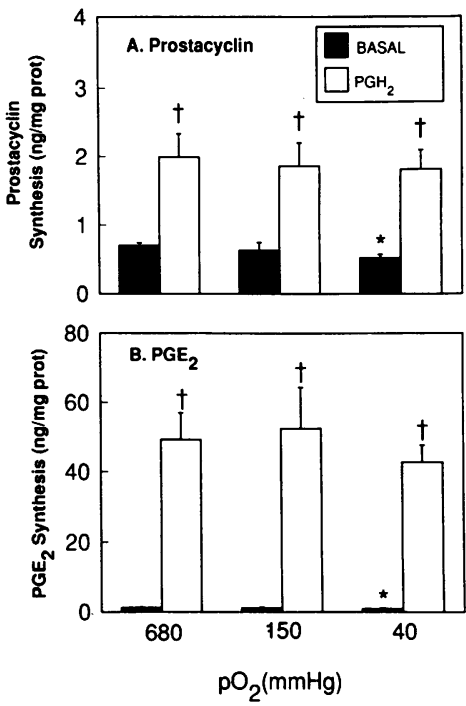

Figure 7. Effect of oxygen tension on $\mathrm{PGH}_{2}$-stimulated $(A)$ prostacyclin and (B) $\mathrm{PGE}_{2}$ synthesis. Pulmonary arterial segments were incubated for $10 \mathrm{~min}$ in the absence of treatment (basal, solid bars) or in the presence of $10^{-5} \mathrm{M} \mathrm{PGH}_{2}$ for $2 \min$ (open bars). The amount of prostacyclin (6keto $\mathrm{PGF}_{1 \propto}$ ) and $\mathrm{PGE}_{2}$ produced was measured by radioimmunoassay. Values are mean \pm SEM for duplicate segments from six fetal lambs. ${ }^{*} P<0.05$ vs. 680 $\mathrm{mmHg},{ }^{\dagger} P<0.05$ vs. basal. parallel, $\mathrm{PGE}_{2}$ synthesis with arachidonic acid was not altered at $150 \mathrm{mmHg}$ but was decreased $31 \%$ at $40 \mathrm{mmHg}$.

The effects of alterations in in vitro oxygenation on prostacyclin and $\mathrm{PGE}_{2}$ synthesis in the presence of exogenous $\mathrm{PGH}_{2}$ are depicted in Fig. 7. Changes in $\mathrm{PO}_{2}$ did not affect the production of either prostanoid when the immediate precursor to their synthesis was added to the incubation.

Basal cyclic AMP production. The role of endogenous prostaglandins in the mediation of basal CAMP production in the intrapulmonary arteries is illustrated in Fig. 8. Over the course of a 20-min incubation with IBMX at $\mathrm{Po}_{2} 680 \mathrm{mmHg}$, cAMP increased by $2.3 \pm 0.2 \mathrm{pmol} / \mathrm{mg}$ protein (mean $\pm \mathrm{SEM}, n=6$ ). Treatment with indomethacin resulted in complete attenuation of basal cAMP production. The addition of either exogenous prostacyclin or $\mathrm{PGE}_{2}$ to indomethacin-treated segments reestablished cAMP production to values that were at least comparable to those measured for basal production (IBMX alone).

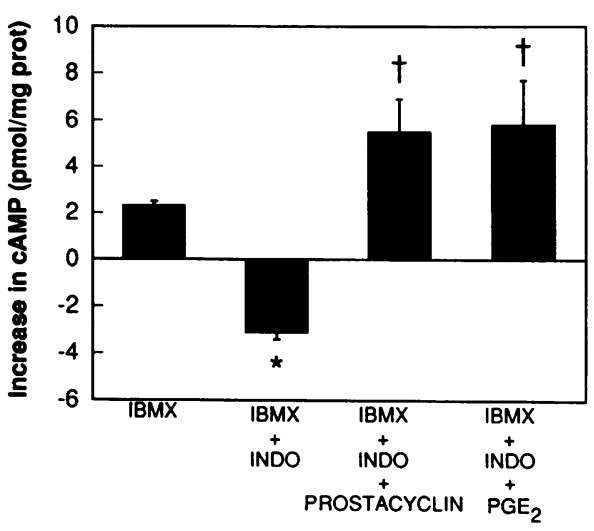

Figure 8. Effect of indomethacin on basal cAMP production. Pulmonary arterial segments were incubated for $10 \mathrm{~min}$ with $10^{-4} \mathrm{M}$ IBMX alone (basal production), or IBMX in the presence of $10^{-4} \mathrm{M}$ indomethacin (INDO), INDO plus $10^{-5} \mathrm{M}$ prostacyclin, or INDO plus $10^{-5} \mathrm{M} \mathrm{PGE}_{2}$. The increase in cAMP content from that in nontreated segments $(7.8 \pm 0.9 \mathrm{pmol} / \mathrm{mg}$ protein $)$ was determined by radioimmunoassay. Values are mean \pm SEM for duplicate segments from four fetal lambs. ${ }^{*} P<0.05$ vs. IBMX alone, ${ }^{\dagger} P<0.05$ vs. IBMX + INDO. 
The effects of alterations in in vitro oxygenation on basal cAMP production in both artery types are depicted in Fig. 9. The cAMP content of nontreated intrapulmonary and systemic arterial segments was similar, at 7.0 \pm 0.7 and 7.2 \pm 0.2 $\mathrm{pmol} / \mathrm{mg}$ of protein, respectively. These values are depicted in Fig. 9 by a single horizontal line. At $\mathrm{Po}_{2} 680 \mathrm{mmHg}$, basal cAMP production yielded a fivefold greater increase in cAMP content in the systemic compared to in the intrapulmonary arteries. With a decline in $\mathrm{PO}_{2}$ to $150 \mathrm{mmHg}$ there was no change in basal cAMP production in either artery type. However, a decline in $\mathrm{Po}_{2}$ to $40 \mathrm{mmHg}$ resulted in complete inhibition of basal cAMP production in the intrapulmonary arteries, and this was contrasted by an increase in the systemic segments of $38 \%$.

Stimulated cyclic AMP production. The effects of changes in oxygenation on prostacyclin-stimulated cAMP production are shown in Fig. $10 \mathrm{~A}$. In studies performed at $\mathrm{Po}_{2} 680 \mathrm{mmHg}$, prostacyclin caused a 2.3-fold increase in cAMP content in the intrapulmonary arteries, and this response was $38 \%$ of that observed in the systemic segments, in which prostacyclin raised cAMP by 6.1 -fold. Cyclic AMP production with stimulation by exogenous prostacyclin was similar at all three levels of oxygenation in both artery types. In addition, in the intrapulmonary segments there was no effect of changing oxygenation on cAMP production stimulated by isoproterenol (Fig. $10 \mathrm{~B}$ ) or forskolin (Fig. $10 \mathrm{C}$ ).

\section{Discussion}

In the present study we have determined the effects of acute changes in in vitro oxygenation on prostacyclin synthesis in intrapulmonary arteries from late gestation ovine fetuses. We have demonstrated that oxygen modulates prostacyclin synthesis, with attenuated synthesis at lower levels of oxygenation. In contrast, we have shown that there is augmented prostacyclin synthesis at lower oxygen tension in comparably sized mesenteric segments. These findings indicate that the oxygen-mediated processes in the intrapulmonary arteries are specific to that vascular bed.

To our knowledge, this is the first time that oxygen-mediated regulation of prostanoid synthesis has been demonstrated in intact intrapulmonary arteries. Previous studies have been primarily performed with cultured cells from large conduit arteries. Rabinovitch et al. (30) demonstrated attenuated prostacyclin and $\mathrm{PGE}_{2}$ synthesis at lower oxygenation $(1 \mathrm{~h})$ in ovine fetal VSM cells from main pulmonary artery, and no effect in aortic VSM. There was a lack of effect on prostaglandin synthesis in endothelial cells from either artery. When pulmonary VSM from newborn lambs was examined, attenuated prostacyclin and $\mathrm{PGE}_{2}$ synthesis were demonstrated at low oxygen tension (30). Our findings in the intrapulmonary arteries are also in agreement with those of Madden et al. (31), who reported decreased prostacyclin synthesis in bovine pulmonary artery endothelial cells exposed to lower oxygen levels for $4 \mathrm{~h}$. In their studies, attenuation of prostacyclin synthesis was also evident in aortic endothelial cells (31). Because pulmonary prostaglandins are primarily produced by the endothelium (16), it is most likely that the effect of alterations in oxygen tension in our experiments was primarily on the endothelial cells. However, based on the findings of Rabinovitch and co-

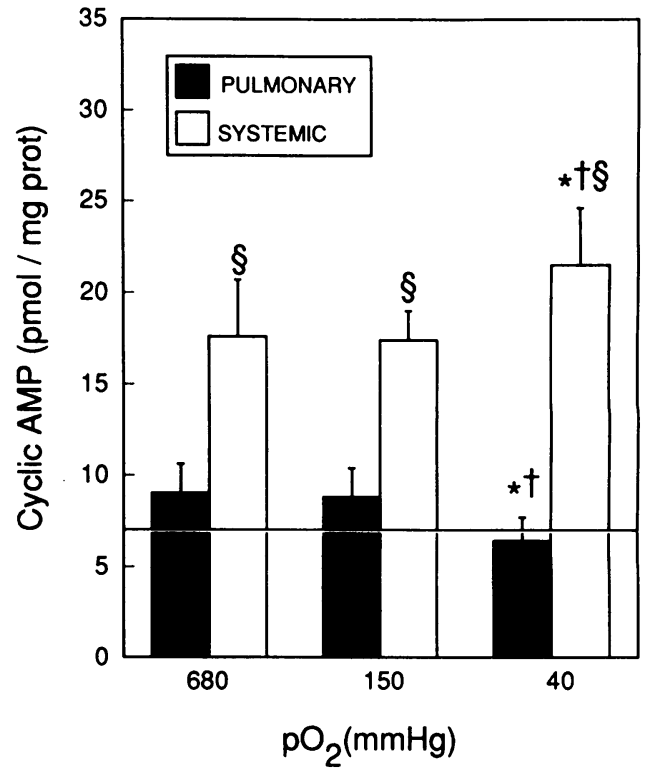

Figure 9. Effect of oxygen tension on basal cAMP production in pulmonary (solid bars) and systemic arterial segments (open bars). Arterial segments were incubated for $10 \mathrm{~min}$ in the presence of $10^{-4} \mathrm{M}$ IBMX and cAMP content was determined by radioimmunoassay. The cAMP content of nontreated pulmonary and systemic arterial segments was similar, at $7.0 \pm 0.7$ and $7.2 \pm 0.2 \mathrm{pmol} / \mathrm{mg}$ protein, respectively; these values are depicted in the figure by a single horizontal line. Values are mean \pm SEM for duplicate segments from 12 fetal lambs. ${ }^{*} P<0.05$ vs. $680 \mathrm{mmHg},{ }^{\dagger} P<0.05$ vs. $150 \mathrm{mmHg},{ }^{\S} P<0.05$ vs. pulmonary.

workers (30), a contribution from the VSM cannot be excluded.

In addition, we have identified the step in the synthetic cascade which is involved in oxygen modulation of prostacyclin synthesis. The first experiments examined the effects of varying oxygenation on agonist-stimulated prostacyclin synthesis with bradykinin. Fineman et al. (25) have proposed that alterations in oxygenation lead to changes in bradykinin production, which in turn modify endothelial prostacyclin synthesis. However, in the present study the $26 \%$ attenuation of basal synthesis at the lowest $\mathrm{Po}_{2}$ was paralleled by $35 \%$ diminution of synthesis with maximally stimulating amounts of bradykinin, indicating that the effect of oxygen is not due to alterations in the production of a local agonist. Alternatively, the effect of oxygen must be more distal in the synthetic cascade.

We next examined the stimulation of prostacyclin synthesis by non-receptor-mediated activation of arachidonic acid mobilization using A23187. Because the degree of attenuation of basal synthesis at the lowest oxygen tension tested was mimicked in the studies with maximally-stimulating concentrations of A23187 (25\% vs. 33\%, respectively), it is apparent that oxygen modulation of prostacyclin synthesis does not involve changes in arachidonic acid mobilization. This conclusion is supported by the finding that the effect of decreased oxygenation is also fully evident when maximally-stimulating concentrations of exogenous arachidonic acid are present (35\% decline). As such, the effect of oxygen must involve alterations in cyclooxygenase and/or prostacyclin synthetase activity.

The possible effects of alterations in oxygenation on the 

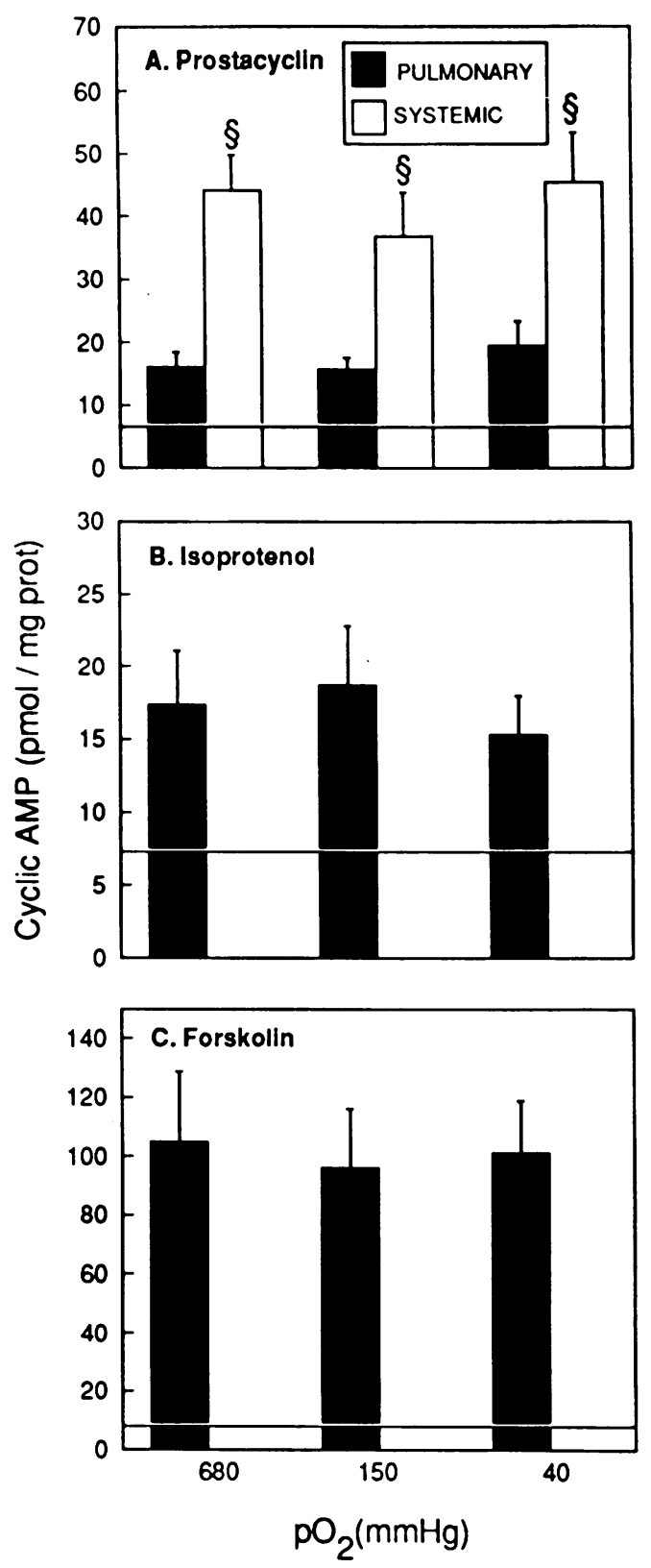

Figure 10. Effect of oxygen tension on stimulated cAMP production in pulmonary ( solid bars) and systemic arterial segments (open bars). Arterial segments were incubated for $10 \mathrm{~min}$ in the presence of $(A)$ $10^{-4} \mathrm{M}$ IBMX plus $10^{-5} \mathrm{M}$ prostacyclin, $(B)$ IBMX plus $10^{-5} \mathrm{M}$ isoproterenol, or $(C)$ IBMX plus $10^{-4} \mathrm{M}$ forskolin. Cyclic AMP content was then determined by radioimmunoassay. The comparable cAMP contents of nontreated pulmonary and systemic arterial segments are depicted in each figure by a single horizontal line. Values are mean \pm SEM for duplicate segments from 12 fetal lambs. Note the differences in the scales in $A, B$, and $C .{ }^{\S} P<0.05$ vs. pulmonary.

activities of these enzymes were differentiated by stimulating prostacyclin synthesis with exogenous $\mathrm{PGH}_{2}$, the product of cyclooxygenase and the substrate of prostacyclin synthetase (26). $\mathrm{PGH}_{2}$-stimulated prostacyclin synthesis was similar at all three levels of oxygenation, indicating that the effect of oxygen is not on prostacyclin synthetase activity. As such, it is concluded that changes in oxygenation modify cyclooxygenase activity. This interpretation is further supported by the observa- tion that basal prostacyclin and $\mathrm{PGE}_{2}$ synthesis, and also prostacyclin and $\mathrm{PGE}_{2}$ synthesis with stimulation proximal to cyclooxygenase, are modulated by oxygen to the same degree.

The activity of cyclooxygenase is dependent not only on the provision of arachidonic acid as substrate, but also on the availability of molecular oxygen, hydroperoxides and heme (32, 33 ). In addition, the rate of enzyme protein synthesis is critical because cyclooxygenase undergoes self-catalyzed inactivation (34), with a half-life less than 10 min in some systems $(35,36)$. It is postulated that oxygen modulation of prostacyclin synthesis in the intrapulmonary arteries is not simply related to changes in the availability of molecular $\mathrm{O}_{2}$. This conclusion is supported by the observation that lowering oxygenation failed to attenuate prostacyclin synthesis in similarly-sized vascular segments from another arterial bed. Furthermore, based on measurements of oxygen consumption by cyclooxygenase in preparations from sheep seminal vesicles, the enzymatic requirement for $\mathrm{O}_{2}$ is far less than the amount available in our studies, even at the lowest oxygen tension tested (32). It is also postulated that hydroperoxides are not involved. Although they are required for the initiation of cyclooxygenase activity, thereafter they paradoxically mediate the autoinactivation of the enzyme (37). As such, at low oxygen tension which yields decreased hydroperoxide levels, prostanoid synthesis over 10 min would actually be expected to be enhanced if hydroperoxides had oxygen-related effects to autoinactivate the enzyme in the intrapulmonary arteries. Instead, it is proposed that oxygen modulation of cyclooxygenase activity may entail alterations in the expression of the enzyme protein or in the availability of its associated prosthetic group, heme.

In addition to demonstrating oxygen modulation of prostacyclin synthesis in the intrapulmonary and systemic arteries, we have determined whether this causes alterations in local cAMP production. We first examined the role of endogenous prostaglandins in the regulation of cAMP production. At $\mathrm{PO}_{2}$ $680 \mathrm{mmHg}$, indomethacin completely attenuated basal cAMP production and it was fully restored by exogenous prostacyclin or $\mathrm{PGE}_{2}$. These data indicate that endogenously synthesized prostaglandins are the primary local mediators of basal cAMP production. We then demonstrated that the attenuation of prostacyclin synthesis in intrapulmonary arteries and the contrasting enhancement in synthesis in systemic arteries with decreased oxygenation are paralleled by diminished and increased basal cAMP production, respectively. Prostacyclin-, isoproterenol-, and forskolin-stimulated cAMP production, however, were not modified by alterations in oxygenation, indicating that oxygen modulation of basal cAMP production is not due to changes in the responsiveness of the adenylate cyclase system. Alternatively, the direct effects of changes in oxygenation on prostacyclin and $\mathrm{PGE}_{2}$ synthesis in both the fetal intrapulmonary and systemic circulation most likely cause the dramatic changes in local cAMP production.

There are important potential physiological ramifications of oxygen modulation of prostacyclin synthesis and cAMP production in both the developing pulmonary and systemic circulation. In the pulmonary circulation, these mechanisms may account at least in part for the decline in pulmonary vascular resistance that occurs in response to increased oxygenation (14 ). This may act in synergy with oxygen modulation of endothelium-derived relaxing factor (or nitric oxide) production, which we have demonstrated results in changes in endothe- 
lium-dependent vasodilatation in vitro (18). Conversely, the enhancement in prostacyclin and $\mathrm{PGE}_{2}$ synthesis in the systemic arteries in response to decreased oxygenation may in part explain the fall in systemic vascular resistance observed with hypoxia in vivo (1-3). Similar mechanisms have been identified by Busse and colleagues in studies of mature canine femoral artery (38). It is further postulated that in tandem, the observed oxygen modulation of vasodilatory prostaglandin synthesis in the two circulations may play a key role in the simultaneous decline in pulmonary vascular resistance and increase in systemic vascular resistance during the normal transition from fetal to postnatal life, resulting in diminution of the right-to-left shunt that is mandatory for existence in utero but contradictory to survival after birth.

There are also potential pathophysiological ramifications of oxygen modulation of prostaglandin synthesis and cAMP production in the developing pulmonary circulation. In addition to having a role in the regulation of vasomotor tone, prostacyclin also attenuates vascular cell growth via cAMP-mediated mechanisms (39). As such, hypoxia-induced declines in prostacyclin synthesis in the pulmonary circulation and the resulting fall in cAMP production may play a key role not only in the vasoconstriction, but also in the medial hypertrophy and other VSM changes that are associated with hypoxic pulmonary hypertension of long duration (40). Meyrick and co-workers (41) have demonstrated that chronic indomethacin treatment in sheep causes sustained pulmonary hypertension, altered pulmonary vasoreactivity and a reduction in pulmonary arterial density, partially mimicking the findings with chronic hypoxic pulmonary hypertension (40). In addition, Rabinovitch et al. (42) have shown that exogenous angiotensin II prevents chronic hypoxic pulmonary hypertension and the associated vascular changes in rats, most likely by inducing prostacyclin production. Furthermore, Badesch and collaborators (43) have demonstrated decreased pulmonary arterial prostacyclin and $\mathrm{PGE}_{2}$ production after severe chronic pulmonary hypertension in neonatal calves. However, the majority of the structural vascular changes accompanying prolonged hypoxia in rats can be prevented by decreasing pulmonary blood flow and pressure (44). Thus, it appears that hypoxia-induced declines in prostacyclin production may at least partially underly chronic hypoxic pulmonary hypertension, and that processes related to changes in hemodynamic stress are probably also involved.

In summary, we have demonstrated that prostacyclin synthesis in ovine fetal intrapulmonary arteries is modulated by alterations in oxygenation, with decreased oxygen tension causing attenuated synthesis. This process is specific to the pulmonary circulation, it is due to an effect on cyclooxygenase activity, and it causes marked parallel alterations in cAMP production. It is concluded that effects of oxygen on prostacyclin synthesis may play an important role in the responses of the developing pulmonary and also systemic circulation to changes in oxygenation, thereby contributing to successful cardiovascular adaptation at birth, as well as to hypoxic pulmonary hypertension in the newborn.

\section{Acknowledgments}

We are indebted to Mr. Kevin M. Horning for his skilled technical assistance and to Ms. Marilyn Dixon for preparing this manuscript.
This work was supported in part by a grant-in-aid from the American Heart Association, Texas Affiliate (No. 90R-113) and by National Institutes of Health grants HL-03791, HD-08783, and HL-24971.

\section{References}

1. Lewis, A. B., M. A. Heymann, and A. M. Rudolph. 1976. Gestational changes in pulmonary vascular responses in fetal lambs in utero. Circ. Res. 39:536-541.

2. Rudolph, A. M. 1979. Fetal and neonatal pulmonary circulation. Annu. Rev. Physiol. 41:383-395.

3. Lyrene, R. K., K. A. Welch, G. Guillermo, and J. B. Philips. 1985. Alkalosis attenuates hypoxic pulmonary vasoconstriction in neonatal lambs. Pediatr. Res. 19:1268-1271.

4. Morin, F. C., E. A. Egan, W. Ferguson, and C. E. G. Lundgren. 1988 Development of pulmonary vascular response to oxygen. Am. J. Physiol. 254:H542-H546.

5. Abman, S. H., F. J. Accurso, R. B. Wilkening, and G. Meschia. 1987. Persistent fetal pulmonary hypoperfusion after acute hypoxia. Am. J. Physiol. 253:H941-H948.

6. Soifer, S. J., D. Kaslow, C. Roman, and M. A. Heymann. 1987. Umbilical cord compression produces pulmonary hypertension in newborn lambs: a model to study the pathophysiology of persistent pulmonary hypertension in the newborn. J. Dev. Physiol. 9:239-252.

7. Fox, W. W., M. H. Gewitz, R. Dinwiddie, W. H. Drummond, and G. J. Peckham. 1977. Pulmonary hypertension in the perinatal aspiration syndromes. Pediatrics. 59:205-211.

8. Dworetz, A. R., F. R. Moya, B. Sabo, I. Gladstone, and I. Gross. 1989. Survival of infants with persistent pulmonary hypertension without extracorporeal membrane oxygenation. Pediatrics. 84:1-6.

9. Campbell, A. G. M., F. Cockburn, G. S. Dawes, and J. E. Milligan. 1967. Pulmonary vasoconstriction in asphyxia during cross-circulation between twin foetal lambs. J. Physiol. (Lond.). 192:111-122.

10. Green, R., J. Rojas, and H. Sundell. 1979. Pulmonary vascular response to prostacyclin in fetal lambs. Prostaglandins. 18:927-934.

11. Lock, J. E., P. M. Olley, and F. Coceani. 1980. Direct pulmonary vascular responses to prostaglandins in the conscious newborn lamb. Am. J. Physiol. 238:H631-H638.

12. Leffler, C. W., and J. R. Hessler. 1981. Perinatal pulmonary prostaglandin production. Am. J. Physiol. 241:H756-H759.

13. Leffler, C. W., J. R. Hessler, and R. S. Green. 1984. The onset of breathing at birth stimulates pulmonary vascular prostacyclin synthesis. Pediatr. Res. 18:938-942.

14. Davidson, D. 1988. Pulmonary hemodynamics at birth: effects of acute cyclooxygenase inhibition in lambs. J. Appl. Physiol. 64:1676-1682.

15. Leffler, C. W., T. L. Tyler, and S. Cassin. 1978. Effect of indomethacin on pulmonary vascular response to ventilation of fetal goats. Am. J. Physiol. 235:H346-H351.

16. Gryglewski, R. J., R. Korbut, and A. Ocetkiewicz. 1978. Generation of prostacyclin by lungs in vivo and its release into the circulation. Nature (Lond.). 273:765-767.

17. Shaul, P. W., M. A. Farrar, and L. M. Buja. 1991. Ontogeny of beta-adrenergic regulation of adenylate cyclase in intrapulmonary arteries from fetal and postnatal lambs. Pediatr. Res. 30:610-615.

18. Shaul, P. W., M. A. Farrar, and T. M. Zellers. 1992. Oxygen modulates endothelium-derived relaxing factor production in fetal pulmonary arteries. $\mathrm{Am}$ J. Physiol. 262:H355-H364.

19. Levin, D. L., A. M. Rudolph, M. A. Heymann, and R. H. Phipps. 1976. Morphological development of the pulmonary vascular bed in fetal lambs. Circulation. 53:144-151.

20. Tripp, M. E., M. A. Heymann, and A. M. Rudolph. 1978. Hemodynamic effects of prostaglandin $\mathrm{E}_{1}$ on lambs in utero. Adv. Prostaglandin Thromboxane Res. 4:221-229.

21. Rudolph, A. M., and M. A. Heymann. 1978. Hemodynamic changes induced by blockers of prostaglandin synthesis in the fetal lamb in utero. Adv. Prostaglandin Thromboxane Res. 4:231-237.

22. Magness, R. R., K. Osei-Boaten, M. D. Mitchell, and C. R. Rosenfeld. 1985. In vitro prostacyclin production by ovine uterine and systemic arteries: effects of angiotensin II. J. Clin. Invest. 76:2206-2212.

23. Lowry, O. H., N. J. Rosebrough, A. L. Farr, and R. J. Randall. 1951. Protein measurement with the folin phenol reagent. J. Biol. Chem. 193:265-275.

24. McIntyre, T. M., G. A. Zimmerman, K. Satoh, and S. M. Prescott. 1985. Cultured endothelial cells synthesize both platelet-activating factor and prostacyclin in response to histamine, bradykinin, and adenosine triphosphate. J. Clin. Invest. 76:271-280.

25. Fineman, J. R., S. J. Soifer, and M. A. Heymann. 1991. The role of 
pulmonary vascular endothelium in perinatal pulmonary circulatory regulation. Semin. Perinatol. 15:58-62.

26. Smith, W. L. 1986. Prostaglandin biosynthesis and its compartmentation in vascular smooth muscle and endothelial cells. Annu. Rev. Physiol. 48:251-262.

27. Shaul, P. W., B. Kinane, M. A. Farrar, L. M. Buja, and R. R. Magness 1991. Prostacyclin production and mediation of adenylate cyclase activity in the pulmonary artery: alterations after prolonged hypoxia in the rat. J. Clin. Invest 88:447-455.

28. Dembinska-Kiec, A., A. Rucker, and P. S. Schonhofer. 1980. Effects of $\mathrm{PGI}_{2}$ and $\mathrm{PGI}$ analogs on cAMP levels in cultured endothelial and smooth muscle cells derived from bovine arteries. Naunyn-Schmiedeberg's Arch. Pharmacol. 311:67-70.

29. Harper, J. F., and G. J. Brooker. 1975. Femtomolar sensitive radioimmunoassay for cAMP and CGMP after 2' 0 acetylation by acetic anhydride in aqueous solution. J. Cyclic Nucleotide Res. 1:207-218.

30. Rabinovitch, M., N. Boudreau, G. Vella, F. Coceani, and P. M. Olley. 1989. Oxygen-related prostaglandin synthesis in ductus arteriosus and other vascular cells. Pediatr. Res. 26:330-335.

31. Madden, M. C., R. L. Vender, and M. Friedman. 1986. Effect of hypoxia on prostacyclin production in cultured pulmonary artery endothelium. Prostaglandins. 31:1049-1062.

32. Van Der Ouderaa, F. J., M. Buytenhek, D. H. Nugteren, and D. A. Van Dorp. 1977. Purification and characterisation of prostaglandin enderoperoxide synthetase from sheep vesicular glands. Biochim: Biophys. Acta. 487:315-331.

33. Ohki, S., N. Ogino, S. Yamamoto, and O. Hayaishi. 1979. Prostaglandin hydroperoxidase, an integral part of prostaglandin endoperoxide synthetase from bovine vesicular gland microsomes. J. Biol. Chem. 254:829-836.

34. Marshall, P. J., R. J. Kulmacz, and W. E. M. Lands. 1987. Constraints on prostaglandin biosynthesis in tissues. J. Biol. Chem. 262:3510-3517.
35. Wu, K. K., H. Hatzakis, S. S. Los, D. C. Seong, S. K. Sanduja, and H. H Tai. 1988. Stimulation of de novo synthesis of prostaglandin G/H synthase in human endothelial cells by phorbol ester. J. Biol. Chem. 263:19043-19047.

36. Fagan, J., and A. L. Goldberg. 1986. Inhibitors of protein and RNA synthesis cause a rapid block in prostaglandin production at the prostaglandin synthase step. Proc. Natl. Acad. Sci. USA. 83:2771-2775.

37. Kulmacz, R. J., and W. E. M. Lands. 1983. Characteristics of prostaglandin H synthase. Adv. Prostaglandin Thromboxane Leukotriene Res. 11:93-97.

38. Busse, R., U. Forstermann, H. Matsuda, and U. Pohl. 1984. The role of prostaglandins in the endothelium-mediated vasodilatory response to hypoxia. Pflügers Arch. Eur. J. Physiol. 401:77-83.

39. Owen, N. E. 1985. Prostacyclin can inhibit DNA synthesis in vascular smooth muscle cells. In Prostaglandins, Leukotrienes and Lipoxins. J. M. Bailey, editor. Plenum Press, New York. 193-204.

40. Rabinovitch, M., W. Gamble, A. S. Nadas, O. S. Miettinen, and L. Reid. 1979. Rat pulmonary circulation after chronic hypoxia: hemodynamic and structural features. Am. J. Physiol. 236:H818-H827.

41. Meyrick, B., M. E. Niedermeyer, M. L. Ogletree, and K. L. Brigham. 1985. Pulmonary hypertension and increased vasoreactivity caused by repeated indomethacin in sheep. J. Appl. Physiol. 59:443-452.

42. Rabinovitch, M., M. Mullen, H. C. Rosenberg, K. Maruyama, H. O'Brodovich, and P. M. Olley. 1988. Angiotensin II prevents hypoxic pulmonary hypertension and vascular changes in rat. Am. J. Physiol. 254:H500-H508.

43. Badesch, D. B., E. C. Orton, L. M. Zapp, J. Y. Wescott, J. Hester, N. F. Voelkel, and K. R. Stenmark. 1989. Decreased arterial wall prostaglandin production in neonatal calves with severe chronic pulmonary hypertension. $\mathrm{Am}$. J. Respir. Cell. Mol. Biol. 1:489-498.

44. Rabinovitch, M., M. A. Konstam, W. J. Gamble, N. Papanicolaou, M. J. Aronovitz, S. Treves, and L. Reid. 1983. Changes in pulmonary blood flow affect vascular response to chronic hypoxia in rats. Circ. Res. 52:432-441. 